\title{
Epigenetic silencing of microRNA-373 plays an important role in regulating cell proliferation in colon cancer
}

\author{
TAKESHI TANAKA ${ }^{1}$, MAKOTO ARAI ${ }^{1}$, SHUANG WU $^{1}$, TATSUO KANDA ${ }^{1}$, HIDEAKI MIYAUCHI $^{2}$, \\ FUMIO IMAZEKI $^{1}$, HISAHIRO MATSUBARA ${ }^{2}$ and OSAMU YOKOSUKA ${ }^{1}$ \\ Departments of ${ }^{1}$ Medicine and Clinical Oncology, and ${ }^{2}$ Frontier Surgery, \\ Graduate School of Medicine, Chiba University, Chiba 260-8670, Japan
}

Received June 20, 2011; Accepted July 11, 2011

DOI: $10.3892 /$ or.2011.1401

\begin{abstract}
RNAs (miRNA) are non-coding RNAs that negatively control gene expression by cleaving or inhibiting the translation of target gene mRNAs. We used a microarray-based transcriptomic analysis to identify miRNA expression levels that changed in response to epigenetic factors. Specifically, we searched for increased expression of miRNAs prepared from colon cancer cell line DLD-1 after a 96-h treatment with $5 \mu \mathrm{M}$ of 5-aza-2'-deoxycytidine (DAC). Among those identified, transient transfection of miRNA hsa-miR-373 resulted in cytostasis. In addition, bisulfate sequence analysis of the promoter regions of these miRNAs showed aberrant methylation in the cancer cells. In clinical colon samples, hsa-miR-373 was down-regulated in colon cancers $(29 / 40,72.5 \%)$ relative to control samples, whereas the purported oncogene RAB22A (a target gene of hsa-miR-373) was up-regulated (24/40, 60\%). Using methylation-specific PCR, we also observed aberrant methylation of hsa-miR-373 in colon cancers $(35 / 40,87.5 \%)$ relative to controls $(8 / 40,20 \%)$. Based on these results, we conclude that expression of hsa-miR-373 is down-regulated by aberrant methylation in colon cancer and that this miRNA may function by regulating expression of the oncogene RAB22A.
\end{abstract}

\section{Introduction}

microRNAs (miRNA) are small, non-coding RNAs that regulate gene expression by base pairing specifically with the 3'-untranslated sequences of target mRNAs or by directing mRNA degradation (1). miRNAs play important roles in cell proliferation, apoptosis and differentiation $(2,3)$. Previous studies have demonstrated changes in the expression levels of specific miRNAs in tumors relative to normal tissues (4), suggesting that miRNAs function as tumor suppressors or oncogenes in human cancers. In support of this hypothesis, overexpression of three miRNAs (miR-15, miR-16 and

Correspondence to: Dr Makoto Arai, Department of Medicine and Clinical Oncology (K1), Graduate School of Medicine, Chiba University, Inohana 1-8-1, Chiba 260-8670, Japan

E-mail: araim-cib@umin.ac.jp

Key words: microRNA, aberrant methylation, colon cancer
miR-127) has been shown to lead to suppression of tumor cell growth via down-regulation of respective target genes (BCL2 and BCL6) and induction of apoptosis $(5,6)$. In contrast, overexpression of miR-184 has been reported to be oncogenic in squamous cell carcinoma of the tongue (7). Hypermethylation of CpG sites within the promoter regions of miRNAs is known to play an important role in silencing tumor-suppressive miRNAs in cancers (8). CpG islands are targets for epigenetic phenomena associated with altered chromatin structure, transcriptional repression and cancer development $(9,10)$. In this study, we used microRNA array analyses to screen for miRNAs up-regulated in response to treatment of colon cancer cell lines with 5-aza-2'-deoxycytidine (DAC). Our goal was to investigate epigenetic regulation of miRNA expression and to document miRNA inhibitory effects on cell proliferation via down-regulation of suspected oncogenic target genes.

\section{Materials and methods}

Cell lines and DAC treatment. Colon cancer cell lines (HT-29, DLD-1 and Colo201) were provided by the Japanese Health Science Research Resources Bank. Colon cancer cell lines (LoVo, WiDr and HCT116) were provided by the American Type Culture Collection (ATCC). HT-29, DLD-1 and Colo201 cells were maintained in RPMI-1640 medium supplemented with $10 \%$ fetal bovine serum (FBS; Sanko Junyaku Co., Ltd., Tokyo, Japan). LoVo cells were maintained in Ham's F12 medium supplemented with $20 \%$ FBS. WiDr cells were maintained in Dulbecco's modified Eagle's medium supplemented with $10 \%$ FBS. HCT-116 cells were maintained in McCoy's 5A medium supplemented with 10\% FBS. Cell lines were treated with DAC (Sigma-Aldrich Co., St. Louis, MO, USA) dissolved in medium just before use. Cells were grown in medium containing 0 (control), 1,2 , or $5 \mu \mathrm{M}$ DAC for $96 \mathrm{~h}$, with replacement of fresh medium and the drug every $24 \mathrm{~h}$. Cell viability after the 96-h DAC treatment was determined by using 3-(4,5-dimethylthiazol-2-yl)-5-(3-carboxymethoxyphenly)-2-(4-sulfonyl)-2H-tetrazolium (MTS) assays in triplicate (CellTiter 96 AQueous One Solution cell proliferation assay; Promega Corp., Madison, WI, USA).

Extraction of DNA and RNA from cell lines. Total RNA was prepared using TRIzol reagent (Invitrogen Co., Carlsbad, CA, 
USA) and DNA was extracted through the use of a QIAamp DNA mini kit (Qiagen Co., Hilden, Germany) according to the manufacturer's instructions. miRNAs were isolated from $60 \mu \mathrm{g}$ of total RNA using the flashPAGE ${ }^{\mathrm{TM}}$ Fractionator system and reagents (Ambion Inc., Austin, TX, USA).

Microarray analysis. We analyzed the mirVana ${ }^{\mathrm{TM}}$ miRNA Bioarray V2, containing 662 miRNAs (Ambion), to identify microRNAs subject to aberrant methylation. Purified miRNA was labeled using Ambion's mirVana ${ }^{\mathrm{TM}}$ miRNA labeling kit and amine-reactive dyes, as recommended by the manufacturer. Labeled miRNAs were hybridized to the array for $\sim 16 \mathrm{~h}$ at $42^{\circ} \mathrm{C}$, after which the hybridized array was washed, dried, and subject to a high-resolution scan using a GenePix 4000B (Axon Instruments, CA, USA). Signal data from each array were subject to global normalization and quantified using the Array-Pro Analyzer Version 4.5 (Media Cybernetics, Inc., Silver Spring, MD, USA).

Evaluation of DAC-induced changes in miRNA expression levels using quantitative RT-PCR. Ten nanograms of total RNA from DAC-treated or untreated cell lines was reverse transcribed using the TaqMan Reverse Transcription kit (Applied Biosystems, Foster City, CA, USA) according to the manufacturer's protocol. Quantitative RT-PCR for the 10 miRNAs (including miR-517a, $-517 \mathrm{~b},-372,-373,-519 \mathrm{~d}$ and $-520 \mathrm{~g}$ ) was carried out using the Taqman MicroRNA assay (Applied Biosystems). For normalization, each miRNA was amplified on the same plate with the reference miRNA, RNU6B, and changes in expression levels were calculated relative to this standard.

Cell viability after transient transfection of miRNAs in cancer cell lines. Pre-miR miRNA precursor molecules $(10 \mathrm{nM})$ that mimic miR-517a, miR-517b miR-372, miR-373, miR-519d, miR-520g and control non-specific miRNA (pre-miR negative control) were obtained from Ambion Co. Using siPORT NeoFX (Ambion), we transfected each miRNA into the cells (HT-29, DLD-1, LoVo and WiDr) grown in medium without FBS, and the transfection medium was then replaced with fresh medium plus FBS after 24 and $48 \mathrm{~h}$. We measured cell viability based on the average of 6 MTS assays per sample (quantified by absorbance at $490 \mathrm{~nm}$ in a microplate colorimeter).

Identifying transcription start sites of microRNAs. We used the SMART ${ }^{\mathrm{TM}}$ RACE cDNA Amplification kit (Clontech, Laboratories, Inc.) to locate the transcription start site (TSS) of each microRNA according to the manufacturer's instructions. cDNA amplification products were also subcloned using the TOPO TA cloning sequencing kit (Invitrogen). CpG Island Searcher (http://www.cpgsilands.com) was then used to search each gene for $\mathrm{CpG}$ sequence islands within 500 base pairs (bp) upstream of each miRNA TSS (lower limits of selection: \%GC, 55\%; observed $\mathrm{CpG} /$ expected $\mathrm{CpG}, 0.65$; length, $200 \mathrm{bp}$; distance, $100 \mathrm{bp}$ ).

Bisulfite sodium modification. Extracted DNA $(1 \mu \mathrm{g})$ was modified with bisulfite using the EpiTect ${ }^{\circledR}$ Bisulfite kit (Qiagen) according to the manufacturer's instructions. Bisulfite genomic sequencing analysis was also used to clarify the methylation status of the $5^{\prime} \mathrm{CpG}$ islands upstream of miR-373 and $-520 \mathrm{~g}$. Bisulfite-modified gene promoters were amplified from genomic DNA $(0.04 \mu \mathrm{g})$ using the following PCR primers and annealing temperature: 1st PCR for miR-373, forward 5'-GAGTGTTATTGTTTGAGAAG-3' and reverse 5'-CCTCATAATCCACCTACCTC- 3 ' at $50^{\circ} \mathrm{C}$; 2nd PCR, forward 5'-GAGGGGGTGGGTAGTAGGATGG-3' and reverse 5'-CTTCCTTTCACCATCTAACCC-3' at $45^{\circ} \mathrm{C}$. 1st PCR for miR-520g, forward 5'-GGTAATATA TTTGTAGAGTGTG-3' and reverse 5'-CCTAAATTAC AACACTACAC- $3^{\prime}$ at $50^{\circ} \mathrm{C}$; 2nd PCR, forward $5^{\prime}$-AGA TTTGTATGATGTTGGGG-3' and reverse 5'-TACAACA CTACACTCCAACC $-3^{\prime}$ at $45^{\circ} \mathrm{C}$. Temperature and times were as follows: pre-incubation for $15 \mathrm{~min}$ at $95^{\circ} \mathrm{C}$, followed by 40 cycles at $95^{\circ} \mathrm{C}$ for $30 \mathrm{sec}$, at the annealing temperature for $30 \mathrm{sec}$, and then $72^{\circ} \mathrm{C}$ for $30 \mathrm{sec}$. Amplified DNAs were subcloned into TA-cloning vector (Invitrogen), and the number of methylated cytosine residues in the promoter from 15 independent colonies was determined for each transformation.

Primer sets for methylation-specific PCR amplification. Aberrant methylation patterns and demethylation in response to DAC treatment were evaluated by methylation- specific PCR (MSP) using the following oligonucleotide primers and annealing temperatures: miR-373 methylated, forward 5'-AAGTGTTGCGATATTTGAGC-3' and reverse 5'-CAA TTTCCGACTAAAAATCGA-3'; unmethylated, forward 5'-GGAAAGTGTTGTGATATTTGAGT-3' and reverse 5'-CAATTTCCAACTAAAAATCAAAAC- $3^{\prime}$ at $45^{\circ} \mathrm{C}$; $\mathrm{miR}$ 520g methylated, forward 5'-TGAGAGCGTTTTTATTTC-3' and reverse 5'-ACACGATAACGAACATCTCT-3'; unmethylated, forward 5'-TTTTGAGATGTGTTTTTATTTT-3' and reverse 5'-ACACAATAACAAACATCTCTATT- 3 ' at $45^{\circ} \mathrm{C}$. PCR products of miR-373 or $-520 \mathrm{~g}$ were estimated from -773 to -660 or from -819 to -679 bp upstream of each coding region in the genome.

Promoter assay. $\mathrm{CpG}$ sequence island within 500 base pairs (bp) upstream of miR-373 TSS was amplified by PCR and then cloned into PCR2.1-TOPO (Invitrogen). Each PCR primer carried 5' overhang that contained $K p n \mathrm{I}$ and $\mathrm{XhoI}$ recognition sites, so that after verifying the sequences, fragments could be cut using KpnI and XhoI and ligated into pGL3-Basic vector (Promega). Cells ( $5 \times 10^{4}$ per well in 6 -well plates) were transfected with $400 \mathrm{ng}$ of one of the reporter plasmids using Effectene Transfection reagent (Qiagen). pGL3-Basic vector without an insert served as the negative control. Luciferase activities were measured $48 \mathrm{~h}$ after transfection using a DualLuciferase Reporter Assay system (Promega).

Primer sequences and annealing temperature were forward 5'-GCTCATCAAGGGCTACTCTC-3' and reverse 5'-CCCTCTGAACCTTCTCTTCC-3' at $45^{\circ} \mathrm{C}$.

Expression levels of miRNA target genes after transfection or DAC treatment of the cell lines. Colon cancer cell lines (HT-29 and DLD-1) were transfected with pre-miR miRNA precursor molecules mimicking miR-373, $-520 \mathrm{~g}$ or the control nonspecific miRNA at a concentration of $200 \mathrm{pM}$ using siPORT 
Table I. miRNA expression levels in DLD-1 cells after DAC treatment.

\begin{tabular}{lrrrrr}
\hline & \multicolumn{2}{c}{$\begin{array}{c}\text { Microarray } \\
\text { (ratio) }\end{array}$} & & \multicolumn{2}{c}{$\begin{array}{c}\text { Real-time } \\
\text { PCR (ratio) }\end{array}$} \\
\cline { 2 - 3 } \cline { 6 - 7 } & \multicolumn{2}{c}{ DAC } & & \multicolumn{2}{c}{ DAC } \\
\cline { 2 - 3 } \cline { 5 - 6 } miRNA & $1 \mu \mathrm{M}$ & $5 \mu \mathrm{M}$ & & $1 \mu \mathrm{M}$ & $5 \mu \mathrm{M}$ \\
\hline miR-517b & 14.4 & 10.7 & & 137.7 & 1118.2 \\
miR-517a & 11.7 & 9.1 & & 986.8 & 1359.1 \\
miR-512-3p & 5.4 & 6.1 & & 1.0 & 1.2 \\
miR-519d & 6.2 & 5.7 & & 75.5 & 85.3 \\
miR-520g & 6.2 & 5.4 & & 1121.8 & 1325.9 \\
miR-126 & 0.5 & 4.9 & & 0.2 & 0.3 \\
miR-373 & 5.1 & 4.3 & & 30.8 & 106.3 \\
miR-372 & 6 & 4.3 & & 172.3 & 184 \\
miR-122a & 0.1 & 4.1 & & 0.4 & 0.4 \\
miR-516-5p & 3.4 & 3.4 & & 0.1 & 0.2 \\
\hline
\end{tabular}

DAC, 5-Aza-CdR. The number of up-regulated miRNAs in DLD-1 cells following 1 and $5 \mu \mathrm{M}$ DAC treatment, respectively, was 51 and 61.

NeoFX. RNA was extracted after a 48-h transfection, cDNA was synthesized using SuperScript III First-Strand Synthesis SuperMix (Invitrogen), and predicted targets gene for miR-373 and miR-520g were identified using TargetScan (http://www. targetscan.org). Among the 10 most likely target genes identified, we focused on the cancer-related genes $R A B 22 A$ (miR-373) and $K L H L 2$ (miR-520g). Steady-state expression levels of $R A B 22 A$ and $K L H L 2$ mRNAs were evaluated by quantitative RT-PCR using Fast SYBR Green master mix and the following oligonucleotide primers and annealing temperatures: $R A B 22 A$, forward 5'-TCGGGGATACAGGTGTAGG-3' and reverse 5'-TTGGTACTGGACAGTCTTGG-3' at $60^{\circ} \mathrm{C}$; KLHL2, forward 5'-CAAGGATGTGAGGCAAGAG-3' and reverse 5'-AGCACTGCTATTCTTGACC-3' at $60^{\circ} \mathrm{C}$. Expression of each gene was quantified relative to a $\beta$-actin control (forward 5'-GACAGGATGCAGAAGGAGATTACT-3' and reverse 5'-TGATCCACATCTGATGGAAGGT-3').

siRNA transfection. siRNAs complementary to each target gene (RAB22A and KLHL2) (Applied Biosystems, Tokyo, Japan) or a control were transfected into HT-29 or DLD-1 cells (plated to reach $80 \%$ confluency after 48 or $72 \mathrm{~h}$ ) using siPORT NeoFX. Medium was replaced every $24 \mathrm{~h}$, and cell viability was quantified from the average of six MTS readings per sample measured at $490 \mathrm{~nm}$ with a microplate colorimeter.

Patient samples. This study was approved by the Ethics Committee of Chiba University, and written informed consent was obtained from all patients. Colorectal cancer samples and appropriately matched controls were obtained from surgical specimens immediately following resection from 40 patients [age, 64.3 \pm 15.8 years; gender (M/F), 20/20] undergoing
Table II. Expression levels of miRNAs following DAC treatment in the cell lines.

\begin{tabular}{lrrrr}
\hline & \multicolumn{3}{c}{ Real-time PCR (ratio) in cell lines: } \\
\cline { 2 - 5 } miRNA & \multicolumn{1}{c}{ LoVo } & WiDr & HCT-116 & Colo201 \\
\hline miR-517b & 1159.7 & 974.4 & 1461.3 & 79.4 \\
miR-517a & 30.7 & 698.3 & 1094.1 & 266.1 \\
miR-372 & 124.3 & 7.4 & 286.4 & 147.9 \\
miR-373 & 118.1 & 6.6 & 562.3 & 269.4 \\
miR-519d & 309.2 & 51.3 & 184.8 & 41.2 \\
miR-520g & 561.8 & 1832 & 1018.2 & 13.4 \\
\hline
\end{tabular}

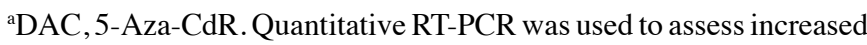
expression levels of miR-517b, $-517 \mathrm{a},-372,-373,-519 \mathrm{~d}$ and $-520 \mathrm{~g}$ in LoVo, WiDr, HCT-116 and Colo201 cell lines.

surgery for colorectal cancer. The samples were preserved in RNA Later ${ }^{\circledR}$ (Ambion) for $24 \mathrm{~h}$ at $4^{\circ} \mathrm{C}$, and then stored at $-80^{\circ} \mathrm{C}$. Total RNA was extracted from surgical specimens using the same method as for the colon cancer cell lines, and the expression levels of miR-373 and $-520 \mathrm{~g}$ and their target genes, $R A B 22 A$ and $K L H L 2$, in cancer specimens were examined relative to normal tissues using quantitative RT-PCR. To analyze changes in methylation state, we performed MSP of bisulfite-modified genomic DNA obtained from surgical specimens using the same primers as for the colon cancer cell line analyses.

Immunohistochemistry. Pieces of colorectal cancer were fixed and stained with hematoxylin and eosin for histological examination. Immunohistochemistry (IHC) was performed on some slides using anti-RAB22A polyclonal antibody (Proteintech Group Inc., Chicago, IL, USA; diluted 1:50). Positively stained cells were visualized using Vectastain ABC Elite kit (Vector Laboratories, Burlingame, CA) and counterstained with Mayer's hematoxylin.

\section{Results}

miRNA expression levels following DAC treatment. Using microarray analysis to detect changes in miRNA expression levels after DAC treatment, we observed between 51 and 61 DAC-dependent increases in specific miRNAs following treatments with 1 and $5 \mu \mathrm{M}$ DAC in DLD- 1 cells, respectively. The top 10 miRNAs demonstrating the most significant increases in expression following $5 \mu \mathrm{M}$ DAC treatment are listed and the expression levels were analyzed using quantitative RT-PCR (Table I). Six miRNAs were up-regulated in this cell line, as well as in five other cell lines (HT-29, LoVo, WiDr, HCT-116 and Colo201). These mRNAs included miR-517a, $-517 \mathrm{~b},-372$, $-373,-519 \mathrm{~d}$ (chromosome 19q13.41) and -520g (chromosome 19q13.42) (Table II).

Effect of miRNA transient transfection on cell proliferation. Four colon cancer cell lines (HT-29, DLD-1, LoVo and WiDr) 

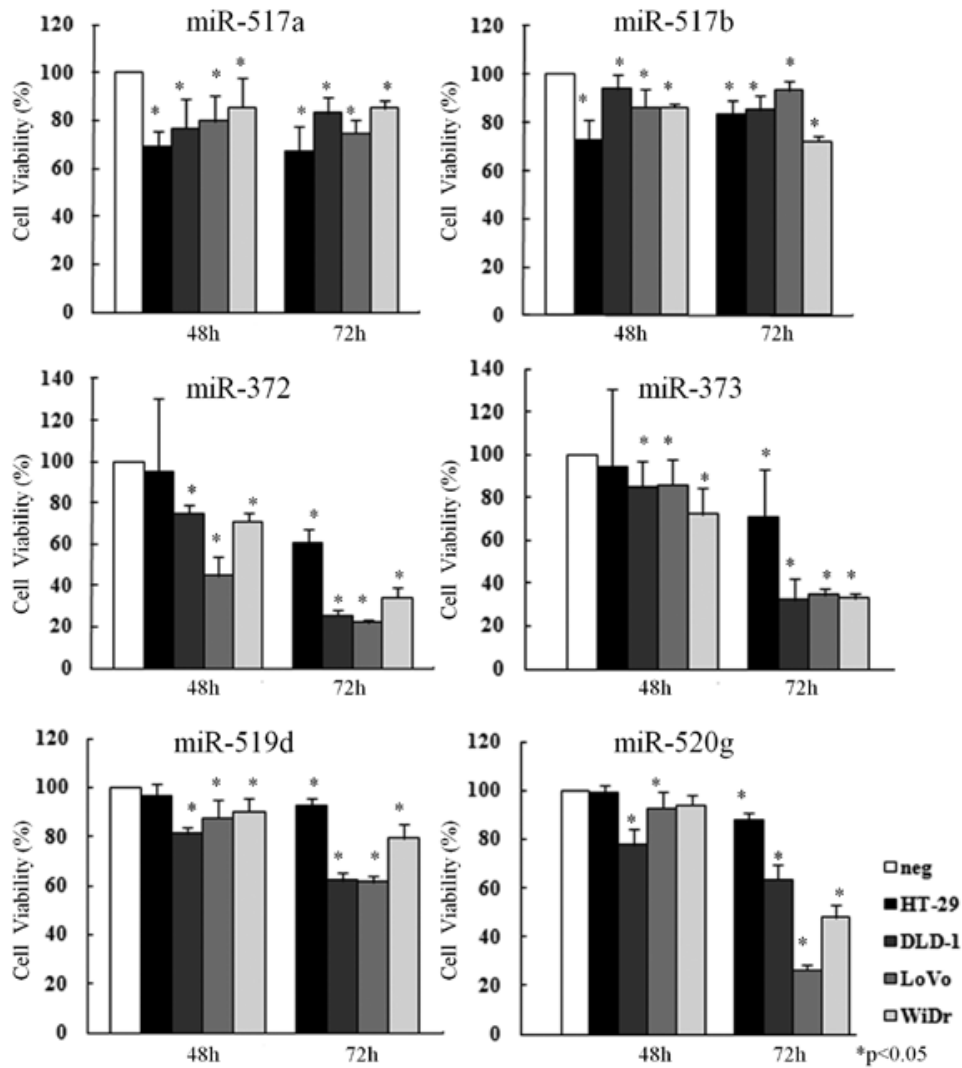

Figure 1. Cell proliferation in colon cancer cell lines (HT-29, DLD-1, LoVo and WiDr) following transient transfection with miRNAs (miR-517a, -517b, -372, $-373,-519 \mathrm{~d}$ and $-520 \mathrm{~g}$ ). White bars indicate cell proliferation in mock-transfected control cells of each line, while shaded bars indicate the viability of transfected cells in each line. Significant differences in cell proliferation were observed in most cancerous cell lines relative to the control ("p $<0.05$, unpaired t-test).



Figure 2. Methylation state of miR-373 and miR-520g promoter regions in colon cancer cell lines. Bisulfite genomic sequencing was performed to assess the methylation of CpG islands of miR-373 and $-520 \mathrm{~g}$ in the HT-29 and DLD-1 cell lines. Methylation state of 14 CpG dinucleotides located between -327 and +13 in miR-373 and 12 CpG dinucleotides located between -661 and -341 in the promoter of miR-520g. Open circles, non-methylated; filled circles, methylated; dashed lines mark $\mathrm{CpG}$ island regions, whereas black-filled arrows mark binding sites for bisulfite sequence primers. White arrows mark primer binding sites for methylation-specific PCR (MSP). Gray arrows mark primer binding sites for promoter assay. 


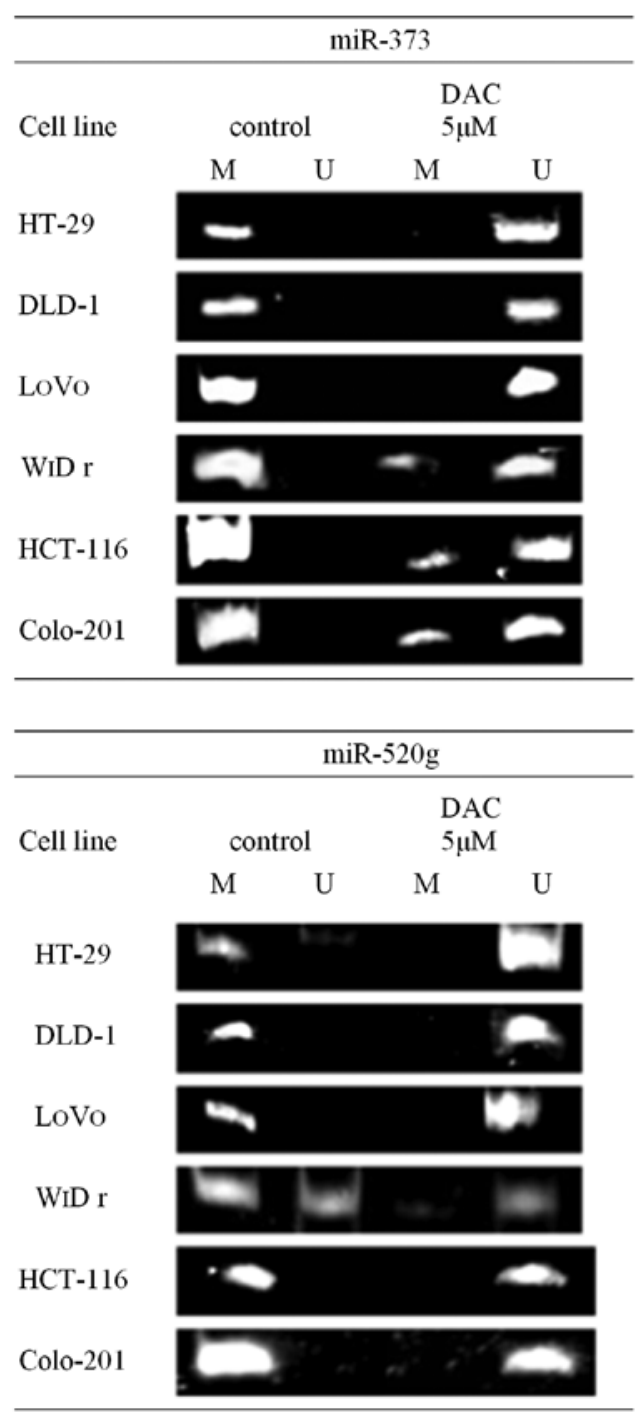

Figure 3. Primer sets of methylation-specific PCR (MSP) amplification. Aberrant methylation and subsequent demethylation of miR-373 and $-520 \mathrm{~g}$ before and after DAC treatment.

were transfected with miR-517a, $-517 \mathrm{~b},-372,-373,-519 \mathrm{~d}$ and $-520 \mathrm{~g}$ precursors or a negative control. MTS assays at 48 and $72 \mathrm{~h}$ post-transfection were used to assess growth inhibition. Relative to the negative control, most experimental miRNAs (517a, 517b, 372, 373, 519d and 520g) significantly inhibited cell proliferation in four colon cancer cell lines $(\mathrm{p}<0.05$, unpaired t-test) (Fig. 1).

Locating miRNA transcription start sites and CpG islands. Transcription start sites for five miRNAs (miR-517a, -372, $-373,-519 \mathrm{~d}$ and $-520 \mathrm{~g}$ ) were estimated at $-431,-917,-569$, -370 and -275 bp upstream of each coding region in the genome; the TSS of miR-517b was not determined due to technical difficulties. Among the five upstream regions sequenced, only two (miR-373 and 520g) showed evidence of $\mathrm{CpG}$ islands. We determined the methylation status of $\mathrm{CpG}$ islands in the promoter regions of miR-373 and $-520 \mathrm{~g}$. Using bisulfite genomic sequencing analysis, aberrant methylation was observed in the promoter regions of miR-373 and $-520 \mathrm{~g}$ in the HT-29 and DLD-1 cells (Fig. 2). Both

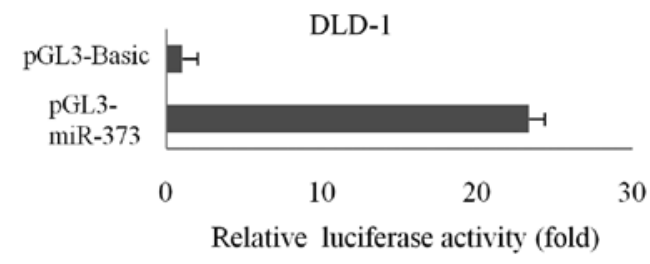

Figure 4. Promoter assay of miR-373 in DLD-1 cells. Results of the promoter assay of miR-373 in the colon cancer cell line DLD-1. Normalized luciferase activities are represented. Columns, means of three replications; error bars, SDs.
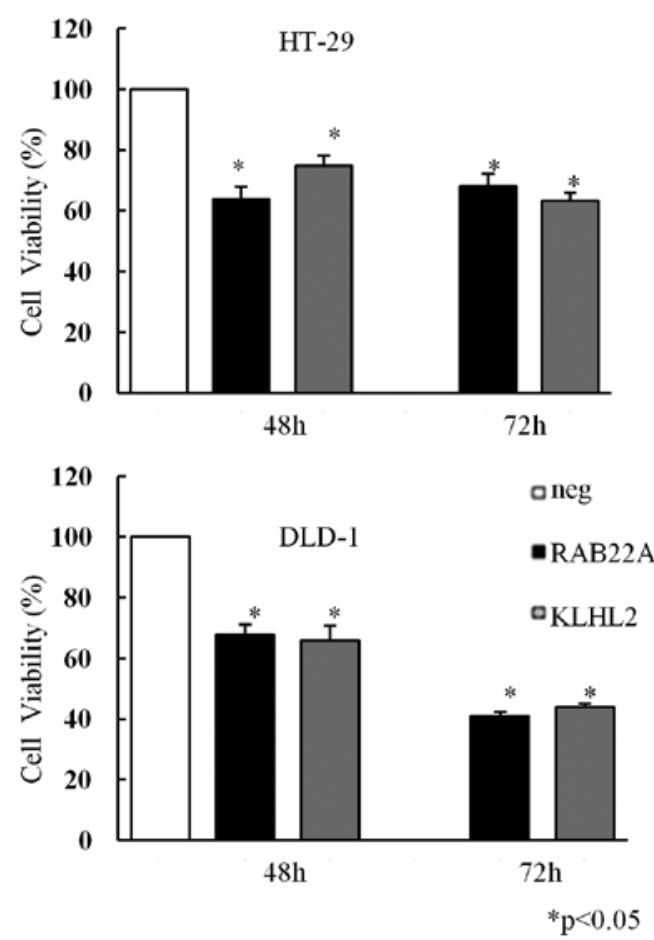

Figure 5. Cell proliferation after transient transfection with siRNA (RAB22A or KLHL2) in HT-29 and DLD-1 cells. White bars indicate cell proliferation in transfected control siRNA, while black bars or shaded bars indicate the cell viability of transfected RAB22A or KLHL 2 siRNA. Significant suppression of cell proliferation was observed in colon cancer cell lines HT29 and DLD1 relative to the control ( ${ }^{*} \mathrm{p}<0.05$, unpaired t-test).

miR-373 and -520g showed aberrant methylation patterns and hypomethylation in response to DAC treatment in all six cell lines (Fig. 3).

Promoter assay. To confirm that the promoter region was located in the $\mathrm{CpG}$ sequence island within 500 bp upstream of miR-373 TSS, we constructed a reporter vector containing the predicted promoter sequences. After transient transfection of colon cancer cell line DLD-1, we observed high levels of luciferase activity with pGL3-miR373 (Fig. 4).

Quantitative RT-PCR of miRNA target genes following miRNA transfection or DAC treatment of the cell lines. Quantitative RT-PCR showed that transient transfection of each miRNA led to a significant suppression of $R A B 22 A$ and KLHL2 expression in HT-29 and DLD-1 cells relative to the control-transfected cells. Each ratio of RAB22A and 
Table III. Expression levels of miR-373, -520g and target genes, and results of the methylation-specific PCR and immunohistochemistry of RAB22A in tissues from 40 colorectal cancer patients.

\begin{tabular}{|c|c|c|c|c|c|c|c|c|c|}
\hline & \multicolumn{3}{|c|}{$\operatorname{miR}-373$} & \multicolumn{2}{|c|}{ RAB22A } & \multicolumn{3}{|c|}{ miR-520g } & \multirow[t]{2}{*}{ KLHL2 } \\
\hline & \multicolumn{3}{|c|}{ Aberrant methylation } & \multirow[b]{2}{*}{$\begin{array}{l}\text { Up- } \\
\text { regulation }\end{array}$} & \multirow[b]{2}{*}{$\begin{array}{l}\text { Immunohisto- } \\
\text { chemistry }\end{array}$} & \multirow[b]{2}{*}{$\begin{array}{l}\text { Down- } \\
\text { regulation }\end{array}$} & \multicolumn{2}{|c|}{ Aberrant methylation } & \\
\hline & $\begin{array}{l}\text { Down- } \\
\text { regulation }^{\mathrm{a}}\end{array}$ & Tumor & $\begin{array}{l}\text { Non- } \\
\text { tumor }\end{array}$ & & & & Tumor & $\begin{array}{l}\text { Non- } \\
\text { tumor }\end{array}$ & $\begin{array}{l}\mathrm{Up}^{\mathrm{b}} \\
\text { regulation }\end{array}$ \\
\hline Total $(n=40)$ & $29(72.5 \%)$ & $35(87.5 \%)^{\mathrm{c}}$ & $8(20 \%)$ & $24(60 \%)$ & $27(67.5 \%)$ & $8(20 \%)$ & $21(52.5 \%)$ & $16(40 \%)$ & $18(45 \%)$ \\
\hline \multicolumn{10}{|l|}{ Location } \\
\hline $\mathrm{A} / \mathrm{C}(\mathrm{n}=8)$ & 5 & 6 & 0 & 5 & 6 & 1 & 4 & 3 & 2 \\
\hline $\mathrm{T} / \mathrm{C}(\mathrm{n}=2)$ & 2 & 2 & 0 & 2 & 2 & 0 & 2 & 1 & 0 \\
\hline $\mathrm{D} / \mathrm{C}(\mathrm{n}=0)$ & 0 & 0 & 0 & 0 & 0 & 0 & 0 & 0 & 0 \\
\hline$S / C(n=16)$ & 12 & 14 & 5 & 9 & 9 & 4 & 8 & 5 & 8 \\
\hline $\operatorname{Rec}(n=14)$ & 10 & 13 & 3 & 8 & 10 & 3 & 7 & 7 & 8 \\
\hline \multicolumn{10}{|l|}{ Stage } \\
\hline $0(n=1)$ & 1 & 1 & 0 & 0 & 0 & 0 & 1 & 0 & 0 \\
\hline $\mathrm{I}(\mathrm{n}=9)$ & 5 & 6 & 2 & 3 & 7 & 1 & 4 & 5 & 4 \\
\hline II $(n=15)$ & 10 & 15 & 4 & 8 & 7 & 4 & 7 & 6 & 9 \\
\hline III (n=9) & 9 & 9 & 1 & 9 & 9 & 1 & 6 & 4 & 2 \\
\hline IV $(n=6)$ & 4 & 4 & 1 & 4 & 4 & 2 & 3 & 1 & 3 \\
\hline
\end{tabular}

A/C, ascending colon; T/C, transverse colon; D/C, descending colon; S/C, sigmoid colon; Rec, rectum. a Down-regulation of miRNA was $<0.5$-fold. ${ }^{b}$ Up-regulation of the target gene was $>1.5$-fold. ${ }^{\mathrm{c}} \mathrm{p}<0.01$, Fisher's exact test.

KLHL2 expression was 0.578 and 0.058 in the HT-29, and 0.006 and 0.054 in the DLD-1 cells, respectively. When treated with DAC, $R A B 22 A$ expression was suppressed in the HT-29, DLD-1, LoVo, WiDr and Colo201 cells relative to the untreated cells; the ratios of gene expression were 0.017 $0.572,0.486,0.953$ and 0.506 , respectively. Yet this effect was not noted in the HCT-116 cells (the ratio of gene expression was 1.667). KLHL2 expression was also suppressed following DAC treatment in all six cell lines relative to the untreated cells; ratios of gene expression were $0.349,0.359,0.540,0.541$, 0.985 and 0.151 , respectively.

siRNA transfection. To determine the effect of the reduced $R A B 22 A$ or $K L H L 2$ mRNA levels on cell proliferation, we transfected gene-specific siRNAs or a non-targeting control siRNA into HT-29 or DLD-1 cells and used MTS assays to measure cell proliferation. Transfection of RAB22A or KLHL2 siRNAs led to a significant decrease in proliferation relative to the control-transfected cells ( $<<0.05$, unpaired t-test) (Fig. 5).

Expression of miR-373, $-520 \mathrm{~g}$ and target genes, and aberrant methylation using methylation-specific PCR in colorectal cancer patients. We assessed miRNA and target gene expression levels in the colon cancer tissues of 40 patients (location of cancer and clinical stage by TMN classification are listed in Table III) compared with normal tissues using quantitative RT-PCR. Relative to normal tissues, there was a reduction in the expression levels of miR-373 $(29 / 40,72.5 \%)$ and $-520 \mathrm{~g}$ $(8 / 40,20 \%)$ in tissues from the colorectal cancer patients. In addition, the expression levels of genes targeted by miR-373 or miR-520g were higher in the cancer patients relative to the controls in $60 \%$ (24/40 for $R A B 22 A)$ or $45 \%$ (18/40 for $K L H L 2)$ of those examined. The percent methylation in the promoter regions of miR-373 in colorectal cancer was $87.5 \%$ (35/40) relative to $20 \%(8 / 40)$ in normal tissues, which showed statistical difference $(\mathrm{p}<0.01$, Fisher's exact test). The miR$520 \mathrm{~g}$ promoter was methylated in $52.5 \%$ (21/40) of cancer tissues relative to $40 \%(16 / 40)$ in normal tissues without a statistical difference (Fisher's test) (Table III). Analysis of IHC revealed that the up-regulation of RAB22A protein in colorectal cancer compared to normal tissue was observed in $67.5 \%$ (27/40) of cases, which was almost compatible with the result of the mRNA expression analysis.

\section{Discussion}

The expression of microRNAs, which negatively control gene expression by cleaving or inhibiting the translation of target gene mRNAs, is down-regulated in various diseases including cancer (4). Changes in microRNA expression are important in the initiation and progression of human cancer (11); for example, the expression of miR-9, -34b/c, -124a, $-129,-137$ and -342 is silenced in colorectal cancers (12-15). In several types of cancer, microRNA expression silencing has been found to be due to aberrant $\mathrm{CpG}$ methylation of its promoter (16).

Epigenetic regulation of miRNAs that subsequently downregulate target gene expression and affect cell proliferation demonstrates a new association between miRNAs and cancer. In this study, we evaluated miRNA expression profiles in colon cancer cell lines before and after DAC treatment. In 
an miRNA array displaying 662 miRNAs, we selected 10 miRNAs that showed a greater than 2-fold increased abundance in each of the cell lines following DAC treatment. Among these, miR-373 and 520g became the focus of this study as CpG islands upstream of the TSS were methylated. These results showed that while overexpression of miR-373 and $-520 \mathrm{~g}$ inhibited cell proliferation, miRNA expression was suppressed by aberrant methylation in the colon cancer cell lines. Colon cancer cell lines and clinical samples in this study both showed that miR-373 expression was down-regulated due to aberrant methylation of a $\mathrm{CpG}$ island in the promoter region. To detect whether the promoter region was located in the $\mathrm{CpG}$ sequence island within 500 bp upstream of miR-373 TSS, we constructed a reporter vector containing the predicted promoter sequences. After transient transfection of colon cancer cell line DLD-1, we observed high levels of luciferase activity with pGL3-miR373. In addition, we confirmed that the promoter region was located in the $\mathrm{CpG}$ sequence island within 500 bp upstream of miR-373 TSS, using Web Promoter Scan Service (http://www-bimas.cit.nih.gov/molbio/proscan).

On the other hand, it has been reported that some miRNAs are located in miRNA clusters (17). Six miRNAs which we investigated were also located in two miRNA clusters, respectively. Although there is a possibility that miRNAs in the same cluster share their promoter lesion, we determined each TSS and promoter lesion of miR-372 and -373. Therefore, we suggest that miR-373 was regulated independently. Although one previous study reported similar down-regulation of miR-373 in a malignant tumor (18), Voorhoeve et al (19) and Lee et al (20) reported miR-373 to be a potential oncogene and found that miR-373 neutralized p53-mediated CDK inhibition, possibly through direct inhibition of the expression of the tumor suppressor LATS2 in testicular germ cell tumors and human esophagus cancer, respectively. These differences suggest that miR-373 serves alternative functions in different types of malignant tumors and acts as a tumor suppressor in colorectal cancers. We used computational predictions to identify $R A B 22 A$ as a potential target gene for miR-373. $R A B 22 A$, a member of the RAS oncogene family, has been reported to be an oncogene in malignant melanoma (21). Similar to colon cancers, its expression level was also found to be up-regulated in hepatocellular carcinoma and cholangiohepatoma relative to controls (22). In colon cancer cell lines treated with DAC, miR-373 expression increased whereas $R A B 22 A$ levels decreased. The expression level of $R A B 22 A$ was also reduced by transient transfection with miR-373. These results suggest that $R A B 22 A$ functions as an oncogene suppressed by miR-373. In colon cancer cell lines, miR-520g expression levels declined due to hypermethylation of promoter sites, and cell proliferation was suppressed due to transient transfection with miR-520g. In contrast, miR-520g expression was not suppressed in clinical samples of colorectal cancers relative to normal tissues. Although epigenetic regulation of miR-373 by aberrant methylation also plays an important role in regulating cell proliferation, discrepancies between cell lines and clinical samples require further analysis.

In conclusion, this study demonstrates a strong association between hypermethylation in $\mathrm{CpG}$ islands upstream of the TSS for miRNA-373 and -520g and down-regulation of miRNA expression. Furthermore, these miRNAs have the ability to inhibit cell viability via the down-regulation of the expression of each target gene in colon cancer cell lines. In clinical samples with aberrant methylation of the miR-373 promoter region, miRNA expression levels were reduced and $R A B 22 A$ target gene levels were increased.

\section{Acknowledgements}

The authors would like to thank Mr. Motohisa Tada and Mrs. Satomi Hasegawa for their excellent technical assistance.

\section{References}

1. He L and Hannon GJ: MicroRNAs: small RNAs with a big role in gene regulation. Nat Rev Genet 5: 522-531, 2004.

2. Ambros V: The functions of animal microRNAs. Nature 431: 350-355, 2004

3. Bartel DP: MicroRNAs: genomics, biogenesis, mechanism, and function. Cell 116: 281-297, 2004.

4. Lu J, Getz G, Miska EA, et al: MicroRNA expression profiles classify human cancers. Nature 435: 834-838, 2005.

5. Cimmino A, Calin GA, Fabbri M, et al: miR-15 and miR-16 induce apoptosis by targeting BCL2. Proc Natl Acad Sci USA 102: 13944-13949, 2005.

6. Saito Y, Liang G, Egger G, et al: Specific activation of microRNA127 with downregulation of the proto-oncogene BCL6 by chromatin-modifying drugs in human cancer cells. Cancer Cell 9: 435-443, 2006.

7. Wong TS, Liu XB, Wong BY, Ng RW, Yuen AP and Wei W: Mature miR-184 as potential oncogenic microRNA of squamous cell carcinoma of tongue. Clin Cancer Res 14: 2588-2592, 2008.

8. Balaguer F, Link A Lozano JJ, et al: Epigenetic silencing of miR-137 is an early event in colorectal carcinogenesis. Cancer Res 70: 6609-6618, 2010.

9. Jones PA and Laird PW: Cancer epigenetics comes of age. Nat Genet 21: 163-167, 1999.

10. Baylin SB: Reversal of gene silencing as a therapeutic target for cancer-roles for DNA methylation and its interdigitation with chromatin. Novartis Found Symp 259: 226-237, 2004.

11. Calin GA and Croce CM: MicroRNA signatures in human cancers. Nature 6: 857-566, 2006.

12. Bandres E, Agirre X, Bitarte N, et al: Epigenetic regulation of microRNA expression in colorectal cancer. Int J Cancer 125: 2737-2743, 2009

13. Toyota M, Suzuki H, Sasaki Y, et al: Epigenetic silencing of microRNA-34b/c and B-cell translocation gene 4 is associated with $\mathrm{CpG}$ island methylation in colorectal cancer. Cancer Res 68: 4123-4132, 2008

14. Lujambio A, Ropero S, Ballestar E, et al: Genetic unmasking of an epigenetically silenced microRNA in human cancer cells. Cancer Res 67: 1424-1429, 2007.

15. Grady WM, Parkin RK, Mitchell PS, et al: Epigenetic silencing of the intronic microRNA has-miR-342 and its host gene EVL in colorectal cancer. Oncogene 27: 3880-3888, 2008.

16. Lee KH, Lotterman C, Karikari C, et al: Epigenetic silencing of microRNA miR-107 regulates cyclin-dependent kinase 6 expression in pancreatic cancer. Pancreatology 9: 293-301, 2009.

17. Rippe V, Dittberner L, Lorenz VN, et al: The two stem cell microRNA gene clusters C19MC and miR-371-3 are activated by specific chromosomal rearrangements in a subgroup of thyroid adenomas. PLoS One 5: e9485, 2010.

18. Ju X, Li D, Shi Q, Hou H, Sun N and Shen B: Differential microRNA expression in childhood B-cell precursor acute lymphoblastic leukemia. Pediatr Hematol Oncol 26: 1-10, 2009.

19. Voorhoeve PM, le Sage C, Schrier M, et al: A genetic screen implicates miRNA-372 and miRNA-373 as oncogenes in testicular germ cell tumors. Cell 124: 1169-1181, 2006.

20. Lee KH, Goan YG, Hsiao M, et al: MicroRNA-373 (miR-373) post-transcriptionally regulates large tumor suppressor, homolog2 (LATS2) and stimulates proliferation in human esophageal cancer. Exp Cell Res 315: 2529-2538, 2009.

21. Okamoto I, Pirker C, Remco MB, et al: Seven novel and stable translocations associated with oncogenic gene expression in malignant melanoma. Neoplasia 7: 303-311, 2005.

22. He H, Dai F, Yu L, et al: Identification and characterization of nine novel human small GTPases showing variable expression in liver cancer tissues. Gene Expression 10: 231-242, 2002. 